\title{
Number of Forts in Iterated Logistic Mapping
}

\author{
Kaixuan $Y u^{1}$ and Zhiheng $Y u^{2}$ \\ ${ }^{1}$ Chengdu Radio and TV University, Chengdu, Sichuan 610051, China \\ ${ }^{2}$ Chengdu Technological University, Chengdu, Sichuan 611730, China \\ Correspondence should be addressed to Zhiheng Yu; yuzhiheng9@163.com
}

Received 15 May 2016; Accepted 17 August 2016

Academic Editor: Daniele Fournier-Prunaret

Copyright ( $) 2016 \mathrm{~K}$. Yu and Z. Yu. This is an open access article distributed under the Creative Commons Attribution License, which permits unrestricted use, distribution, and reproduction in any medium, provided the original work is properly cited.

Using the theory of complete discrimination system and the computer algebra system MAPLE V.17, we compute the number of forts for the logistic mapping $f_{\lambda}(x)=\lambda x(1-x)$ on $[0,1]$ parameterized by $\lambda \in(0,4]$. We prove that if $0<\lambda \leq 2$ then the number of forts does not increase under iteration and that if $\lambda>2$ then the number of forts is not bounded under iteration. Furthermore, we focus on the case of $\lambda>2$ and give for each $k=1, \ldots, 7$ some critical values of $\lambda$ for the change of numbers of forts.

\section{Introduction}

Iteration is the act of repeating a process with the aim of approaching a desired goal, target, or result. In mathematical sense, for a fixed integer $n \geq 1$, the $n$th iterate $f^{n}$ of a mapping $f: E \rightarrow E$, where $E$ is a nonempty set, is defined recursively by

$$
f^{k}=f \circ f^{k-1}, \quad f^{0}=\mathrm{id},
$$

where $\circ$ presents the composition of functions and id denote the identity mapping; that is, $\operatorname{id}(x) \equiv x$ for all $x \in E$. Being indispensable in the computer era, iteration brings many interesting but difficult problems to mathematics. Only from one-dimensional case, one can simply notice that an iterate of a linear function of any order remains linear but the degree of a polynomial may increase drastically, which shows that the nonlinear complexity is amplified by iteration.

Actually, in the one-dimensional case, the complexity of nonlinear functions is related to nonmonotonicity. For a continuous nonmonotonic self-mapping $f: I \rightarrow I$, where $I$ is an interval, a point $x_{0} \in I$ is called a monotone point of $f$ if $f$ is strictly monotone in a neighborhood of $x_{0}$; otherwise, $x_{0}$ is called a nonmonotone point or simply a fort of $f$. Obviously, a linear function does not have a fort generically. In 1980s, Zhang and Yang (see [1]) investigated the number of forts for a class of nonmonotonic functions called strictly piecewise monotone functions and simply PM functions, which are selfmapping on a compact interval and have at most finitely many forts each. Let $S(f)$ denote the set of all forts of $f$ and let $N(f)$ denote the cardinality of $S(f)$. It is shown in $[1,2]$ that

$$
\begin{aligned}
0 & =N\left(f^{0}\right) \leq N(f) \leq N\left(f^{2}\right) \leq \cdots \leq N\left(f^{k}\right) \\
& \leq N\left(f^{k+1}\right) \leq \cdots ;
\end{aligned}
$$

that is, the number $N\left(f^{k}\right)$ of forts is nondecreasing as $k$ is increasing. One can similarly prove that (2) also holds for functions defined on the whole $\mathbb{R}$. It is easy to find nonlinear functions whose number of forts, regarded as the damagers of monotonicity, increases rapidly under iteration. Consider the quadratic function

$$
f(x)=4 x(1-x), \quad x \in[0,1],
$$

for example. Computing derivatives of $f^{i}, i=1, \ldots, 5$, and counting the number of real zeros with odd multiplicity for the derivatives $\left(f^{i}\right)^{\prime}$ (as done in [3]), we get $N(f)=1$, $N\left(f^{2}\right)=3, N\left(f^{3}\right)=7, N\left(f^{4}\right)=15$, and $N\left(f^{5}\right)=31$. From the increasing tendency, without continuing the tedious computation, we have the following question: Does $N\left(f^{k}\right)$ have a bound or approach infinity as $k$ tends to $\infty$ ? How can we compute the number of forts for nonmonotonic functions?

Polynomials, a special class of nonmonotonic functions, possess the advantage that each fort of a polynomial of degree 
$\geq 1$ is either a peak or a valley although the notion is not true in general. In this paper, we focus on the family of logistic mappings:

$$
f_{\lambda}(x)=\lambda x(1-x), \quad x \in[0,1],
$$

where $\lambda \in(0,4]$ is a parameter, which is one of the simplest polynomial mappings, and a typical example used to show chaos and some complicated dynamics, for those problems. First of all, we introduce the theory of complete discrimination system (see [4]) and then use it to give a method for the computation of $N(f)$ with $f$ polynomial in Section 2. In Section 3, we employ the method in the computer algebra system MAPLE V.17 for the family of logistic mappings. We prove in Theorem 4 that $N\left(f_{\lambda}^{k}\right)=N\left(f_{\lambda}\right)=1$ for all integer $k \geq 2$ if $0<\lambda \leq 2$ and that $N\left(f_{\lambda}^{k}\right)$ approaches $\infty$ as $k \rightarrow \infty$ if $2<\lambda \leq$ 4. Furthermore, for various choices of $\lambda \in(2,4]$, we compute the number $N\left(f_{\lambda}^{k}\right)$ for each fixed $k=2,3, \ldots, 7$ in Theorem 5 .

\section{Preliminaries}

In general, for polynomial

$$
f(x)=\sum_{i=0}^{n} a_{i} x^{i}
$$

where $n \geq 2$ and $a_{n} \neq 0 . N\left(f^{k}\right), k=1,2, \ldots$, is decided by real zeros of the derivatives $\left(f^{k}\right)^{\prime}$.

Lemma 1 (see [3, Lemma 2.1]). $x_{0}$ is a fort of a real polynomial $f$ if and only if $x_{0}$ is a real zero of the derivative $f^{\prime}$ of odd multiplicity. Moreover, $N(f)$ is odd (resp., even) if the degree $n$ of $f$ is even (resp., odd).

Actually, the above lemma shows how a real zero of the derivative $f^{\prime}$ can be a fort of $f$. Note that $\left(f^{k}\right)^{\prime}=\left(f^{\prime}\right.$ 。 $\left.f^{k-1}\right)\left(f^{\prime} \circ f^{k-2}\right) \cdots\left(f^{\prime} \circ f\right) f^{\prime}$. Then the set of zeros of $\left(f^{k}\right)^{\prime}$ is a union of the set of zeros of $f^{\prime} \circ f^{k-1}$ and the set of zeros of $\left(f^{\prime} \circ f^{k-2}\right) \cdots\left(f^{\prime} \circ f\right) f^{\prime}$. Therefore, in order to know if $f^{k}$ have more forts than $f^{k-1}$, we need to judge if $f^{\prime} \circ f^{k-1}$ have real zeros different from $\left(f^{\prime} \circ f^{k-2}\right) \cdots\left(f^{\prime} \circ f\right) f^{\prime}$ s with odd multiplicities; the following lemma gives the answer.

Lemma 2 (see [3, Lemma 2.5]). Let $G$ and $P$ be real polynomials and $G(x) \neq \equiv$. Then the composition $G \circ P$ and the derivative $P^{\prime}$ do not have a common real zero with odd multiplicity.

Taking $G=f^{\prime}$ and $P=f^{k-1}$, by Lemmas 1 and 2, we see that $\left(f^{k}\right)^{\prime}$ has more real zeros of odd multiplicities than $\left(f^{k-1}\right)^{\prime}$ if and only if $f^{\prime} \circ f^{k-1}$ has real zeros with odd multiplicities. Hence, in the process of computing $N\left(f^{k}\right)$, we only need to find out the number of real zeros for $f^{\prime} \circ f^{k-1}$ with odd multiplicities. For this reason, we first introduce some notations of the theory of complete discrimination system (see $[4,5])$ which will lead us to solve this problem.

Discriminants of polynomials are useful in determining the number of zeros for polynomials. Let $\operatorname{Discr}(f)$ denote the discriminant matrix of the polynomial $f$, which is constructed by the Sylvester matrix of $f$ and $f^{\prime}$ as seen in [4, Definition 1]. For each $\tau=1, \ldots, n$, let $D_{\tau}(f)$ denote the determinant of its submatrix formed by the first $2 i$ rows and the first $2 i$ columns. The $n$-tuple $\left(D_{1}, D_{2}, \ldots, D_{n}\right)$ is called the discriminant sequence of $f$ and the list

$$
\left(\operatorname{sign}\left(D_{1}\right), \operatorname{sign}\left(D_{2}\right), \ldots, \operatorname{sign}\left(D_{n}\right)\right)
$$

is called the sign list of $f$, where $\operatorname{sign}(x)$ is defined to be equal to either 1 if $x>0,0$ if $x=0$, or -1 if $x<0$. Given a sign list $\left(s_{1}, s_{2}, \ldots, s_{n}\right)$ of $f$, we make a new list $\left(\varepsilon_{1}, \varepsilon_{2}, \ldots, \varepsilon_{n}\right)$, called the revised sign list of $f$, in the following regulation:

If $\left(s_{i}, s_{i+1}, \ldots, s_{i+j}\right)$ is a section of the given list such that $s_{i} \neq 0, s_{i+1}=s_{i+2}=\cdots=s_{i+j-1}=0$, and $s_{i+j} \neq 0$, then replace the subsection $\left(s_{i+1}, s_{i+2}, \ldots, s_{i+j-1}\right)$ with $\left(\varepsilon_{i+1}, \varepsilon_{i+2}, \ldots, \varepsilon_{i+j-1}\right)$, where $\varepsilon_{i+r}=(-1)^{[(r+1) / 2]} s_{i}$ for $r=1,2, \ldots, j-1$; otherwise, let $\varepsilon_{\tau}=s_{\tau}$.

The following lemma tells us how to find the number of distinct zeros by means of the revised sign list.

Lemma 3 (see [4, Theorem 1]). Let $f$ be a real polynomial and suppose that the number of the sign changes in the revised sign list of $f$ is $v$. Then the number of pairs of distinct conjugate complex zeros of $f$ equals $v$. Furthermore, if the number of nonvanishing members in the revised sign list is $l$, then $F$ has $l-2 v$ distinct real zeros.

Now, we are ready to apply the above lemmas to detail the process in computing $N\left(f^{k}\right), k=1,2, \ldots$. First of all, we compute $f^{k}$ and $f^{\prime} \circ f^{k-1}$ and the discriminant sequence for $f^{\prime} \circ f^{k-1}$. Secondly, under algebraic relations among coefficients $a_{i}$ 's in the discriminant sequence, discuss the sign of each component of the discriminant sequence and list the sign lists. Then, compute the revised sign lists for $f^{\prime} \circ f^{k-1}$ through the sign lists. According to the revised sign lists, we find out the number of real zeros of $f^{\prime} \circ f^{k-1}$ with odd multiplicities and finally obtain $N\left(f^{k}\right)$.

The above idea can be implemented in the computer algebra system MAPLE V.17, and we will use this method for the logistic mappings up to iteration index $k=7$ in next section.

\section{Number of Forts}

In this section, we first draw a conclusion for the logistic mappings which describe that the numbers of forts can be preserved or approach $\infty$ as $\lambda$ varies under iteration and then compute $N\left(f_{\lambda}^{k}\right)$ for $f_{\lambda}(x)$ up to iteration index $k=7$ with different choice of $\lambda$.

Theorem 4. The logistic mappings $f_{\lambda}$ defined in (4) have $N\left(f_{\lambda}^{k}\right)=N\left(f_{\lambda}\right)=1$ for all integer $k \geq 2$ if and only if $0<\lambda \leq 2$. Otherwise, $N\left(f_{\lambda}^{k}\right)$ approaches $\infty$ as $k \rightarrow \infty$. 
TABLE 1: The number of forts of $f^{i}, i=1,2, \ldots, 7$.

\begin{tabular}{|c|c|c|c|c|c|c|c|}
\hline Ranges of $\lambda$ & $N\left(f_{\lambda}\right)$ & $N\left(f_{\lambda}^{2}\right)$ & $N\left(f_{\lambda}^{3}\right)$ & $N\left(f_{\lambda}^{4}\right)$ & $N\left(f_{\lambda}^{5}\right)$ & $N\left(f_{\lambda}^{6}\right)$ & $N\left(f_{\lambda}^{7}\right)$ \\
\hline$\lambda_{1,0}<\lambda \leq \lambda_{2,1}$ & 1 & 1 & 1 & 1 & 1 & 1 & 1 \\
\hline$\lambda_{2,1}<\lambda \leq \lambda_{3,2}$ & 1 & 3 & 5 & 7 & 9 & 11 & 13 \\
\hline$\lambda_{3,2}<\lambda \leq \lambda_{3,4}$ & 1 & 3 & 7 & 13 & 21 & 31 & 43 \\
\hline$\lambda_{3,4}<\lambda \leq \lambda_{3,6}$ & 1 & 3 & 7 & 13 & 23 & 37 & 57 \\
\hline$\lambda_{3,6}<\lambda \leq \lambda_{3,7}$ & 1 & 3 & 7 & 13 & 23 & 37 & 59 \\
\hline$\lambda_{3,7}<\lambda \leq \lambda_{4,3}$ & 1 & 3 & 7 & 13 & 23 & 39 & 65 \\
\hline$\lambda_{4,3}<\lambda \leq \lambda_{4,9}$ & 1 & 3 & 7 & 15 & 29 & 53 & 93 \\
\hline$\lambda_{4,9}<\lambda \leq \lambda_{4,10}$ & 1 & 3 & 7 & 15 & 29 & 53 & 95 \\
\hline$\lambda_{4,10}<\lambda \leq \lambda_{4,11}$ & 1 & 3 & 7 & 15 & 29 & 55 & 101 \\
\hline$\lambda_{4,11}<\lambda \leq \lambda_{5,5}$ & 1 & 3 & 7 & 15 & 29 & 55 & 103 \\
\hline$\lambda_{5,5}<\lambda \leq \lambda_{5,12}$ & 1 & 3 & 7 & 15 & 31 & 61 & 117 \\
\hline$\lambda_{5,12}<\lambda \leq \lambda_{6,8}$ & 1 & 3 & 7 & 15 & 31 & 61 & 119 \\
\hline$\lambda_{6,8}<\lambda \leq \lambda_{7,13}$ & 1 & 3 & 7 & 15 & 31 & 63 & 125 \\
\hline$\lambda_{7,13}<\lambda \leq 4$ & 1 & 3 & 7 & 15 & 31 & 63 & 127 \\
\hline
\end{tabular}

Proof. In order to obtain the condition for $N\left(f_{\lambda}^{k}\right)=N\left(f_{\lambda}\right)=$ 1 , from the method mentioned in the end of Section 2, we need to compute $N\left(f_{\lambda}^{2}\right)$. Simple computation shows that

$$
f_{\lambda}^{\prime} \circ f_{\lambda}=2 \lambda x^{2}-2 \lambda x+1
$$

Then computing the discriminant sequence of (7), we have

$$
\left(\lambda^{2}, \lambda^{3}(\lambda-2)\right)
$$

From (8), if $0<\lambda<2$, the revised sign list is $(1,-1)$, implying that $f_{\lambda}^{\prime} \circ f_{\lambda}$ has one pair of complex zeros, which leads to $N\left(f_{\lambda}^{2}\right)=1$; if $\lambda=2$, the revised sign list is $(1,0)$, implying that $f_{\lambda}^{\prime} \circ f_{\lambda}$ has a double real zero, which leads to $N\left(f_{\lambda}^{2}\right)=1$; therefore, $N\left(f_{\lambda}\right)=N\left(f_{\lambda}^{k}\right)=1$ if and only if $0<\lambda \leq 2$.

Without loss of generality, we can turn the general form $f_{\lambda}(x)=-\lambda x^{2}+\lambda x$ into

$$
f_{\lambda}(x)=-\lambda\left(x-\frac{1}{2}\right)^{2}+\frac{\lambda}{4}
$$

So the vertex of the parabola is

$$
\left(x_{0}, y_{0}\right):=\left(\frac{1}{2}, \frac{\lambda}{4}\right) .
$$

If $2<\lambda \leq 4$, then $f_{\lambda}$ and a diagonal line intersect at two points $\xi_{1}$ and $\xi_{2}$, where $\xi_{1}<x_{0}<\xi_{2}$. Obviously, $\xi_{1}$ and $\xi_{2}$ are fixed points of $f_{\lambda} ; \xi_{1}=0$ and $\xi_{2}=(\lambda-1) / \lambda$ and $f_{\lambda}$ is strictly increasing on the subinterval $\left[\xi_{1}, x_{0}\right]$. Thus,

$$
\begin{aligned}
\xi_{1}<f_{\lambda}^{-k-1}\left(x_{0}\right)<f_{\lambda}^{-k}\left(x_{0}\right)<x_{0} & \\
& \forall k=1,2, \ldots, \lim _{k \rightarrow+\infty} f_{\lambda}^{-k}\left(x_{0}\right)=\xi_{1} .
\end{aligned}
$$

Since

$$
S\left(f_{\lambda}^{k}\right)=S\left(f_{\lambda}^{k-1}\right) \cup\left\{x \in[0,1]: f_{\lambda}^{k-1} \in S\left(f_{\lambda}\right)\right\},
$$

we get

$$
\begin{aligned}
\mathcal{S} & \left(f_{\lambda}^{k}\right) \backslash \mathcal{S}\left(f_{\lambda}^{k-1}\right)=\mathcal{S}\left(f_{\lambda} \circ f_{\lambda}^{k-1}\right) \backslash \mathcal{S}\left(f_{\lambda}^{k-1}\right) \\
& =\left(\mathcal{S}\left(f_{\lambda}^{k-1}\right)\right. \\
& \left.\cup\left\{x \in[0,1]: f_{\lambda}^{k-1}(x) \in \mathcal{S}\left(f_{\lambda}\right)\right\}\right) \backslash \mathcal{S}\left(f_{\lambda}^{k-1}\right) \\
& =\left\{x \in[0,1]: f_{\lambda}^{k-1}(x) \in \mathcal{S}\left(f_{\lambda}\right)\right\} \backslash \mathcal{S}\left(f_{\lambda}^{k-1}\right) \\
& \neq \emptyset \quad \forall k \in \mathbb{N}^{+},
\end{aligned}
$$

which implies that $N\left(f_{\lambda}^{k}\right)$ approaches $\infty$ as $k \rightarrow \infty$. This completes the proof.

Theorem 4 shows that the number $N\left(f_{\lambda}^{k}\right)$ approaches $\infty$ as $k \rightarrow \infty$ for each fixed $\lambda \in(2,4]$. It is also interesting to see for each fixed $k$ how the number $N\left(f_{\lambda}^{k}\right)$ varies as the parameter $\lambda$ changes in $(2,4]$. The following theorem shows the change of numbers $N\left(f_{\lambda}^{k}\right)$ as $\lambda$ varies for each $k=2, \ldots, 7$ (but larger $k$ can be considered if the computational capacity of our computer is better). It gives a sequence of parameter values at which new forts arise.

Theorem 5. $N\left(f_{\lambda}^{2}\right)=3$ for all $\lambda \in(2,4]$ and $N\left(f_{\lambda}^{3}\right)=5$ and 7 for $\lambda \in\left(2, \lambda_{3,2}\right]$ and $\lambda \in\left(\lambda_{3,2}, 4\right]$, respectively, where $\lambda_{3,2}:=\sqrt{5}+1 \approx 3.236067977$. For more details, with the convenient notations $\lambda_{1,0}:=0$ and $\lambda_{2,1}:=2$, numbers $N\left(f_{\lambda}^{k}\right)$, $k=1,2, \ldots, 7$, are given in Table 1 , where

$$
\begin{aligned}
& \lambda_{4,3} \approx 3.831874056, \\
& \lambda_{3,4} \approx 3.498561699, \\
& \lambda_{5,5} \approx 3.960270127, \\
& \lambda_{3,6} \approx 3.627557530, \\
& \lambda_{3,7} \approx 3.738914913, \\
& \lambda_{6,8} \approx 3.990267047,
\end{aligned}
$$




$$
\begin{aligned}
& \lambda_{4,9} \approx 3.844568792, \\
& \lambda_{4,10} \approx 3.905706470, \\
& \lambda_{4,11} \approx 3.937536445, \\
& \lambda_{5,12} \approx 3.977766422, \\
& \lambda_{7,13} \approx 3.997583118 .
\end{aligned}
$$

Proof. By (8), if $2<\lambda \leq 4$, the revised sign list of $f_{\lambda}^{\prime} \circ f_{\lambda}$ is $(1,1)$, implying that it has two distinct real zeros, which shows that $N\left(f^{2}\right)=3$.

Furthermore, in order to obtain $N\left(f_{\lambda}^{3}\right)$, we compute

$$
\begin{aligned}
f_{\lambda}^{\prime} \circ f_{\lambda}^{2}= & 2 \lambda^{3} x^{4}-4 \lambda^{3} x^{3}+2 \lambda^{3} x^{2}+2 \lambda^{2} x^{2}-2 \lambda^{2} x \\
& +1
\end{aligned}
$$

As shown in Section 2, we give the discriminant sequence for (15):

$$
\begin{aligned}
& \left(\lambda^{6}, \lambda^{11}(\lambda-2), \lambda^{14}(\lambda-2)^{2}, \lambda^{15}\left(\lambda^{2}-2 \lambda-4\right)\right. \\
& \left.\cdot(\lambda-2)^{3}\right) .
\end{aligned}
$$

Then, the revised sign list for (16) is

(i) $(1,1,1,1)$, if $\lambda_{3,2}<\lambda \leq 4$, which implies that $f_{\lambda}^{\prime} \circ f_{\lambda}^{2}$ has 4 distinct simple real zeros;

(ii) $(1,1,1,0)$, if $\lambda=\lambda_{3,2}$, which implies that $f_{\lambda}^{\prime} \circ f_{\lambda}^{2}$ has 3 distinct real zeros, 2 of which are simple zeros and the remaining one is a double zero;

(iii) $(1,1,1,-1)$, if $\lambda_{2,1}<\lambda<\lambda_{3,2}$, which implies that $f_{\lambda}^{\prime}$ 。 $f_{\lambda}^{2}$ has one pair of complex zeros and 2 distinct simple real zeros.

Here $\lambda_{2,1}=2$ and $\lambda_{3,2}=\sqrt{5}+1$, as defined in the theorem. $\lambda_{3,2}$ is the real zero of $g(\lambda):=\lambda^{2}-2 \lambda-4$ in $(2,4]$. By Lemmas 1 and $2, N\left(f_{\lambda}^{3}\right)=5$ if $\lambda_{2,1}<\lambda \leq \lambda_{3,2}$ and $N\left(f_{\lambda}^{3}\right)=7$ if $\lambda_{3,2}<\lambda \leq 4$.

Similarly, compute

$$
\begin{aligned}
f_{\lambda}^{\prime} \circ f_{\lambda}^{3}= & 2 \lambda^{7} x^{8}-8 \lambda^{7} x^{7}+\left(12 \lambda^{7}+4 \lambda^{6}\right) x^{6} \\
& +\left(-8 \lambda^{7}-12 \lambda^{6}\right) x^{5} \\
& +\left(2 \lambda^{7}+12 \lambda^{6}+2 \lambda^{5}+2 \lambda^{4}\right) x^{4} \\
& +\left(-4 \lambda^{6}-4 \lambda^{5}-4 \lambda^{4}\right) x^{3} \\
& +\left(2 \lambda^{5}+2 \lambda^{4}+2 \lambda^{3}\right) x^{2}-2 \lambda^{3} x+1 .
\end{aligned}
$$

Then we obtain the discriminant sequence for $f_{\lambda}^{\prime} \circ f_{\lambda}^{3}$ :

$$
\begin{aligned}
& \left(\lambda^{14}, \lambda^{27}(\lambda-2), \lambda^{38}(\lambda-2)^{2}, \lambda^{47}\left(\lambda^{2}-2 \lambda-4\right)\right. \\
& \cdot(\lambda-2)^{3}, \lambda^{54}\left(\lambda^{2}-2 \lambda-4\right) \\
& \cdot(\lambda-2)^{4}, \lambda^{59}\left(\lambda(\lambda-2)\left(\lambda^{2}-2 \lambda-4\right)-16\right) \\
& \cdot(\lambda-2)^{5}, \lambda^{62}\left(\lambda^{2}-2 \lambda-4\right) \\
& \cdot\left(\lambda(\lambda-2)\left(\lambda^{2}-2 \lambda-4\right)-16\right) \\
& \cdot(\lambda-2)^{6}, \lambda^{63}\left(\lambda(\lambda-2)\left(\lambda^{2}-2 \lambda-4\right)^{2}-64\right) \\
& \left.\cdot\left(\lambda^{2}-2 \lambda-4\right)^{2}(\lambda-2)^{7}\right) .
\end{aligned}
$$

Hence,

(i) for $\lambda_{2,1}<\lambda<\lambda_{3,2}$, the revised sign list for $f_{\lambda}^{\prime} \circ f_{\lambda}^{3}$ is $(1,1,1,-1,-1,-1,1,-1)$, implying that $f_{\lambda}^{\prime} \circ f_{\lambda}^{3}$ has 3 pairs of complex zeros and 2 distinct simple real zeros;

(ii) for $\lambda=\lambda_{3,2}$, the revised sign list for $f_{\lambda}^{\prime} \circ f_{\lambda}^{3}$ is $(1,1,1,-1,-1,-1,0,0)$ and $f_{\lambda}^{\prime} \circ f_{\lambda}^{3}$ can be factorized as

$$
\begin{aligned}
& f_{\lambda}^{\prime} \circ f_{\lambda}^{3}=\frac{1}{64}(13 \sqrt{5}+29) \\
& \cdot\left(32 x^{2}-32 x+4 \sqrt{5}+12 \sqrt{2}-4 \sqrt{10}-4\right) \\
& \cdot\left(x-\frac{1}{2}-\frac{1}{4} \sqrt{6-2 \sqrt{5}+6 \sqrt{2}-2 \sqrt{10}}\right) \\
& \cdot\left(x-\frac{1}{2}+\frac{1}{4} \sqrt{6-2 \sqrt{5}+6 \sqrt{2}-2 \sqrt{10}}\right) \\
& \cdot(4 x-3+\sqrt{5})^{2}(-4 x+1+\sqrt{5})^{2}
\end{aligned}
$$

implying that $f_{\lambda}^{\prime} \circ f_{\lambda}^{3}$ has a pair of complex zeros and 4 distinct real zeros and two of the 4 distinct real zeros are simple and the rest are both double zeros;

(iii) for $\lambda_{3,2}<\lambda<1+(3+2 \sqrt{5})^{1 / 2}$, where $1+(3+2 \sqrt{5})^{1 / 2}$ is the real zero of $h(\lambda):=\lambda(\lambda-2)\left(\lambda^{2}-2 \lambda-4\right)-16$ in $\left(\lambda_{3,2}, 4\right]$, the revised sign list for $f_{\lambda}^{\prime} \circ f_{\lambda}^{3}$ is $(1,1,1,1,1,-1,-1,-1)$, implying that $f_{\lambda}^{\prime} \circ f_{\lambda}^{3}$ has a pair of complex zeros and 6 distinct simple real zeros;

(iv) for $\lambda=1+(3+2 \sqrt{5})^{1 / 2}$, the revised sign list for $f^{\prime} \circ f^{3}$ is $(1,1,1,1,1,-1,-1,-1)$, implying that $f_{\lambda}^{\prime} \circ f_{\lambda}^{3}$ has a pair of complex zeros and 6 distinct simple real zeros;

(v) for $1+(3+2 \sqrt{5})^{1 / 2}<\lambda<\lambda_{4,3}$, where

$$
\begin{aligned}
& \lambda_{4,3} \\
& =\frac{\sqrt{3}}{3}\left(\frac{2(100+12 \sqrt{69})^{2 / 3}+11(100+12 \sqrt{69})^{1 / 3}+8}{(100+12 \sqrt{69})^{1 / 3}}\right)^{1 / 2} \\
& \quad+1 \approx 3.831874056
\end{aligned}
$$


is the real zero of $p(\lambda):=\lambda(\lambda-2)\left(\lambda^{2}-2 \lambda-4\right)^{2}-64$ in $\left(1+(3+2 \sqrt{5})^{1 / 2}, 4\right]$, the revised sign list for $f^{\prime} \circ f^{3}$ is $(1,1,1,1,1,1,1,-1)$, implying that $f_{\lambda}^{\prime} \circ f_{\lambda}^{3}$ has a pair of complex zeros and 6 distinct simple real zeros;

(vi) for $\lambda=\lambda_{4,3}$, the revised sign list for $f_{\lambda}^{\prime} \circ f_{\lambda}^{3}$ is $(1,1,1,1,1,1,1,0)$, implying that $f_{\lambda}^{\prime} \circ f_{\lambda}^{3}$ has 7 distinct real zeros, one of which is a double zero but the rest are all simple;

(vii) for $\lambda_{4,3}<\lambda \leq 4$, the revised sign list for $f_{\lambda}^{\prime} \circ f_{\lambda}^{3}$ is $(1,1,1,1,1,1,1,1)$, implying that $f_{\lambda}^{\prime} \circ f_{\lambda}^{3}$ has 8 distinct simple real zeros.

It follows that $N\left(f_{\lambda}^{4}\right)=7$ if $\lambda_{2,1}<\lambda \leq \lambda_{3,2}, N\left(f_{\lambda}^{4}\right)=13$ if $\lambda_{3,2}<\lambda \leq \lambda_{4,3}$, and $N\left(f_{\lambda}^{4}\right)=15$ if $\lambda_{4,3}<\lambda \leq 4$.

We similarly compute $f_{\lambda}^{\prime} \circ f_{\lambda}^{4}$ and obtain the discriminant sequence

$$
\begin{aligned}
& \left(\lambda^{30}, \lambda^{59}(\lambda-2), \lambda^{86}(\lambda-2)^{2}, \lambda^{111}\left(\lambda^{2}-2 \lambda-4\right)(\lambda\right. \\
& -2)^{3}, \lambda^{134}\left(\lambda^{2}-2 \lambda-4\right)(\lambda-2)^{4}, \lambda^{155}\left(\lambda^{4}-4 \lambda^{3}\right. \\
& +8 \lambda-16)(\lambda-2)^{5}, \lambda^{174}\left(\lambda^{2}-2 \lambda-4\right)\left(\lambda^{4}-4 \lambda^{3}\right. \\
& +8 \lambda-16)(\lambda-2)^{6}, \lambda^{191}\left(\lambda^{6}-6 \lambda^{5}+4 \lambda^{4}+24 \lambda^{3}\right. \\
& \left.-16 \lambda^{2}-32 \lambda-64\right)\left(\lambda^{2}-2 \lambda-4\right)^{2}(\lambda-2)^{7} \\
& \lambda^{206}\left(\lambda^{6}-6 \lambda^{5}+4 \lambda^{4}+24 \lambda^{3}-16 \lambda^{2}-32 \lambda-64\right) \\
& +\left(\lambda^{2}-2 \lambda-4\right)^{2}(\lambda-2)^{8}, \lambda^{219}\left(\lambda^{2}-2 \lambda-4\right)\left(\lambda^{10}\right. \\
& -10 \lambda^{9}+28 \lambda^{8}+16 \lambda^{7}-160 \lambda^{6}+64 \lambda^{5}+192 \lambda^{4} \\
& \left.+384 \lambda^{3}-256 \lambda^{2}-1024 \lambda+1024\right)(\lambda-2)^{9} \\
& \lambda^{230}\left(\lambda^{4}-4 \lambda^{3}+8 \lambda-16\right)\left(\lambda^{10}-10 \lambda^{9}+28 \lambda^{8}\right. \\
& +16 \lambda^{7}-160 \lambda^{6}+64 \lambda^{5}+192 \lambda^{4}+384 \lambda^{3}-256 \lambda^{2} \\
& -1024 \lambda+1024)(\lambda-2)^{10}, \lambda^{239}\left(\lambda^{2}-2 \lambda-4\right)\left(\lambda^{8}\right. \\
& \left.-8 \lambda^{7}+16 \lambda^{6}+16 \lambda^{5}-64 \lambda^{4}+128 \lambda-256\right)\left(\lambda^{4}\right. \\
& \left.-4 \lambda^{3}+8 \lambda-16\right)^{2}(\lambda-2)^{11}, \lambda^{246}\left(\lambda^{8}-8 \lambda^{7}+16 \lambda^{6}\right. \\
& \left.+16 \lambda^{5}-64 \lambda^{4}+128 \lambda-256\right)\left(\lambda^{2}-2 \lambda-4\right)^{2}\left(\lambda^{4}\right. \\
& \left.-4 \lambda^{3}+8 \lambda-16\right)^{2}(\lambda-2)^{12}, \lambda^{251}\left(\lambda^{4}-4 \lambda^{3}+8 \lambda\right. \\
& -16)\left(\lambda^{14}-14 \lambda^{13}+72 \lambda^{12}-136 \lambda^{11}-144 \lambda^{10}\right. \\
& +1088 \lambda^{9}-1600 \lambda^{8}-640 \lambda^{7}+4096 \lambda^{6}-512 \lambda^{5} \\
& \left.-4096 \lambda^{4}-12288 \lambda^{3}+16384 \lambda^{2}+8192 \lambda-16384\right) \\
& +\left(\lambda^{2}-2 \lambda-4\right)^{3}(\lambda-2)^{13}, \lambda^{254}\left(\lambda^{6}-6 \lambda^{5}+4 \lambda^{4}\right. \\
& +10
\end{aligned}
$$

$$
\begin{aligned}
& \left.+24 \lambda^{3}-16 \lambda^{2}-32 \lambda-64\right)\left(\lambda^{14}-14 \lambda^{13}+72 \lambda^{12}\right. \\
& -136 \lambda^{11}-144 \lambda^{10}+1088 \lambda^{9}-1600 \lambda^{8}-640 \lambda^{7} \\
& +4096 \lambda^{6}-512 \lambda^{5}-4096 \lambda^{4}-12288 \lambda^{3}+16384 \lambda^{2} \\
& +8192 \lambda-16384)\left(\lambda^{2}-2 \lambda-4\right)^{4}(\lambda-2)^{14}, \\
& \lambda^{255}\left(\lambda^{12}-12 \lambda^{11}+48 \lambda^{10}-40 \lambda^{9}-192 \lambda^{8}+384 \lambda^{7}\right. \\
& \left.+64 \lambda^{6}-1024 \lambda^{4}-512 \lambda^{3}+2048 \lambda^{2}+4096\right)\left(\lambda^{6}\right. \\
& \left.-6 \lambda^{5}+4 \lambda^{4}+24 \lambda^{3}-16 \lambda^{2}-32 \lambda-64\right)^{2}\left(\lambda^{2}-2 \lambda\right. \\
& \left.-4)^{5}(\lambda-2)^{15}\right) .
\end{aligned}
$$

Similar discussion gives $N\left(f_{\lambda}^{5}\right)$ for various $\lambda$ as shown in Table 1.

It is more complicated to compute discriminant sequences of $f_{\lambda}^{\prime} \circ f_{\lambda}^{5}$ and $f_{\lambda}^{\prime} \circ f_{\lambda}^{6}$ because the two discriminant sequences contain 32 and 64 components, respectively, and the biggest component in the discriminant sequence of $f_{\lambda}^{\prime} \circ f_{\lambda}^{5}$ is a polynomial in the single variable $\lambda$ of degree 1152 with 130 terms. Using a similar discussion as for $f_{\lambda}^{\prime} \circ f_{\lambda}^{k}$, $k=1,2,3$, 4, we obtain parameter values $\lambda_{3,6}, \lambda_{3,7}, \lambda_{6,8}, \lambda_{4,9}$, $\lambda_{4,10}, \lambda_{4,11}, \lambda_{5,12}$, and $\lambda_{7,13}$ as well as the numbers $N\left(f_{\lambda}^{6}\right)$ and $N\left(f_{\lambda}^{7}\right)$ on intervals between them as shown in Table 1. This completes the proof.

Although we are not able to compute for all $k$ those parameter values for changes of $N\left(f_{\lambda}^{k}\right)$ in Theorem 5, those data of Table 1 for $k=1,2, \ldots, 7$ show that the number $N\left(f_{\lambda}^{k}\right)$ can reach its maximum $2^{k}-1$ if $\lambda>\lambda_{k, l}, l=0,1,2,3,5,8,13$. We naturally have the following.

Question 1. Does $N\left(f_{\lambda}^{k}\right)$ reach the maximum $2^{k}-1$ for any integer $k \geq 1$ ?

Additionally, the well-known Feigenbaum sequence (see $[6,7])$ is $\lambda_{1}=3, \lambda_{2} \approx 3.449490, \lambda_{3} \approx 3.544090, \lambda_{4} \approx$ $3.564407, \lambda_{5} \approx 3.568750, \lambda_{6}=3.569690, \ldots$, at each of which a period-doubling bifurcation happens in the logistic mapping. This suggests the following.

Question 2. Is there any relation between our sequence $\left\{\lambda_{k, l}\right\}$ and the Feigenbaum sequence $\left\{\lambda_{k}\right\}$ ?

A related work can be found from [8], but the question is not answered yet.

\section{Competing Interests}

The authors declare that they have no competing interests.

\section{Acknowledgments}

This work is supported by FP7-PEOPLE-2012-IRSES-316338. 


\section{References}

[1] J. Zhang and L. Yang, "Discussion on iterative roots of piecewise monotone functions, in Chinese," Acta Mathematica Sinica, vol. 26, pp. 398-412, 1983.

[2] W. Zhang, "PM functions, their characteristic intervals and iterative roots," Annales Polonici Mathematici, vol. 65, no. 2, pp. 119-128, 1997.

[3] L. L. Yang, L. Yang, Z. Yu, and W. Zhang, "Real polynomial iterative roots in the case of nonmonotonicity height $\geq 2$," Science China Mathematics, vol. 55, no. 12, pp. 2433-2446, 2012.

[4] L. Yang, X. Hou, and Z. Zeng, "A complete discrimination system for polynomials," Science China E, vol. 39, no. 6, pp. 628646, 1996.

[5] I. M. Gelfand, M. M. Kapranov, and A. V. Zelevinsky, Discriminants, Resultants, and Multidimensional Determinants, Mathematics: Theory \& Applications, Birkhäuser, Boston, Mass, USA, 1994.

[6] K. T. Alligood, T. D. Sauer, and J. A. Yorke, Chaos: An Introduction to Dynamical Systems, Springer, New York, NY, USA, 1996.

[7] R. D. Smith, "Period doubling, information entropy, and estimates for Feigenbaum's constants," International Journal of Bifurcation and Chaos, vol. 23, no. 11, Article ID 1350190, 2013.

[8] G. Linage, F. Montoya, A. Sarmiento, K. Showalter, and P. Parmananda, "Fibonacci order in the period-doubling cascade to chaos," Physics Letters A, vol. 359, no. 6, pp. 638-639, 2006. 


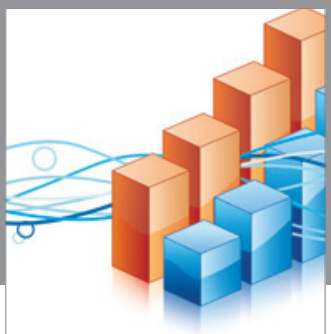

Advances in

Operations Research

vatem alat4

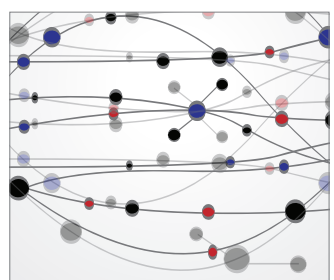

\section{The Scientific} World Journal
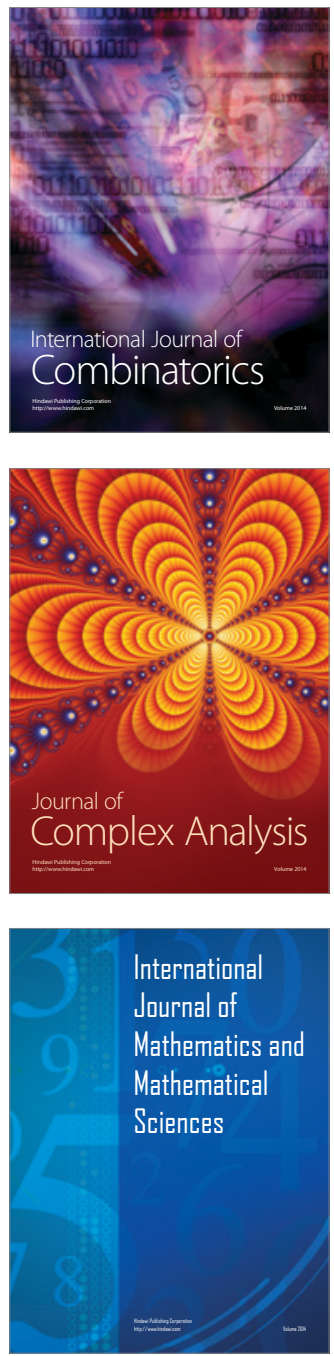
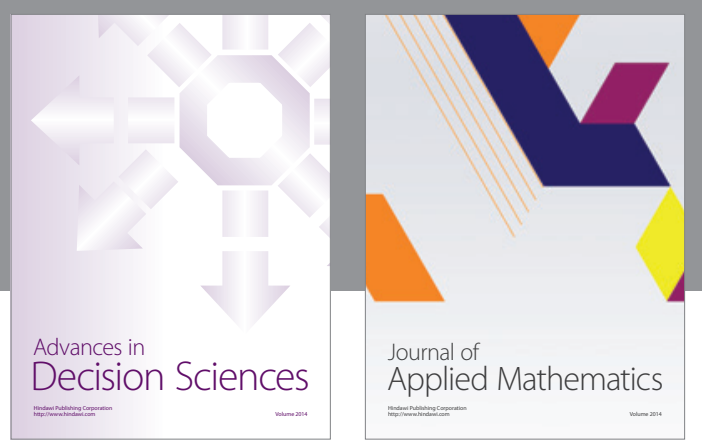

Algebra

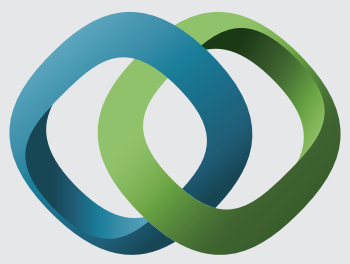

\section{Hindawi}

Submit your manuscripts at

http://www.hindawi.com
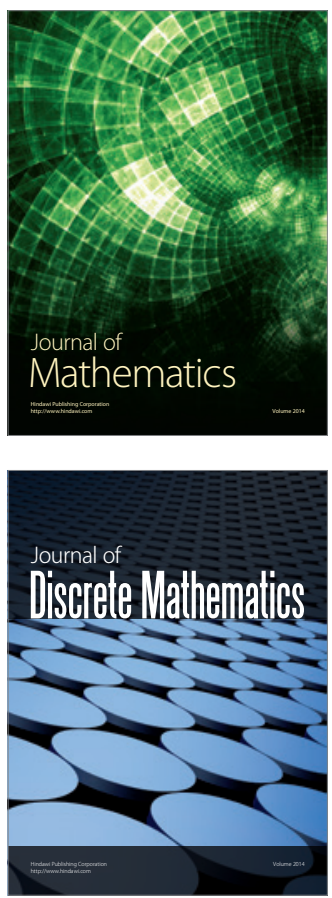

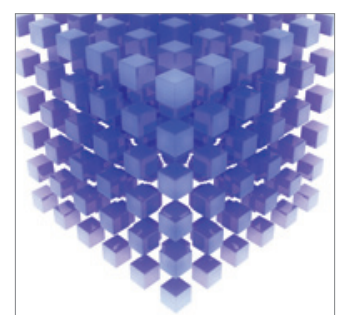

Mathematical Problems in Engineering
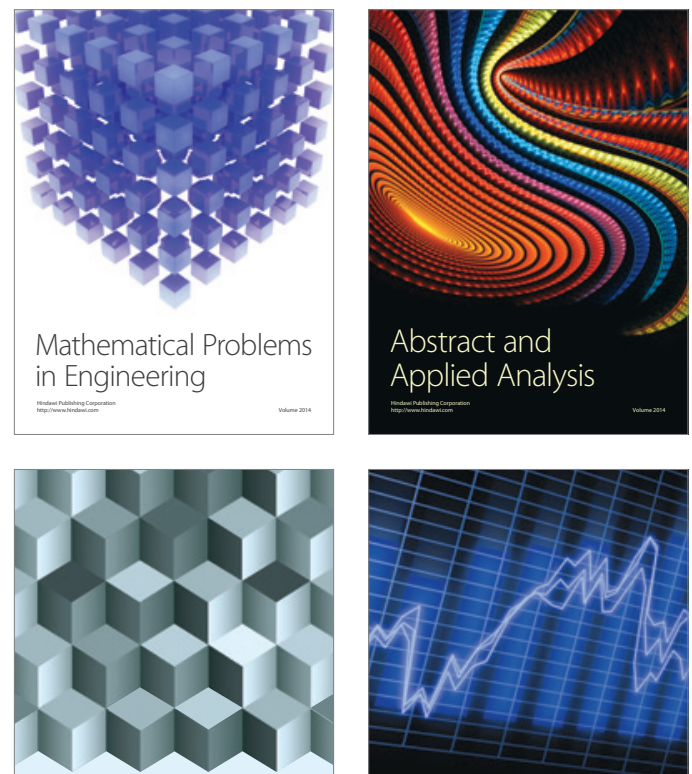

Journal of

Function Spaces

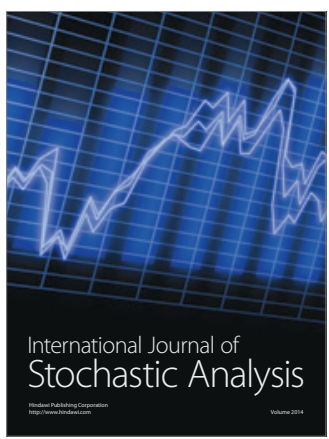

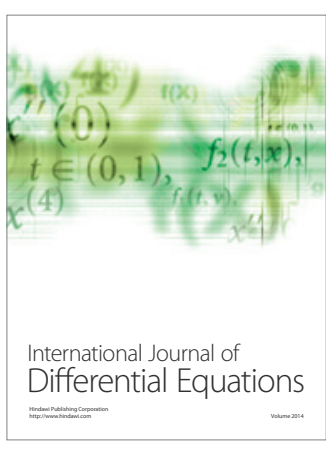
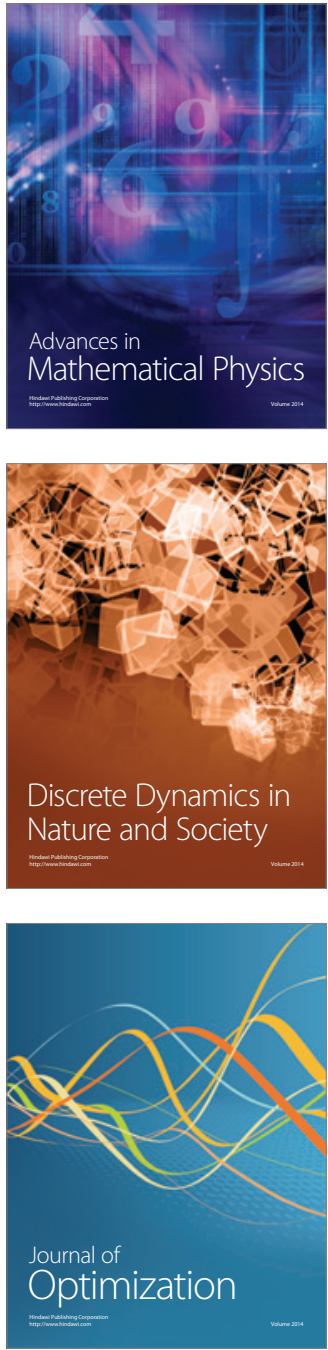